\title{
Quality of life in patients with acromegaly receiving lanreotide autogel: a real-world observational study
}

\author{
Marek Bolanowski', Alicja Hubalewska-Dydejczyk², Beata Kos-Kudła ${ }^{3}$, Marek Ruchała ${ }^{4}$, \\ Przemysław Witek ${ }^{5}$, Wojciech Zgliczyński ${ }^{6}$, Aude Houchard ${ }^{7}$, Marta Bartmańska $^{8}$
}

\author{
${ }^{1}$ Department of Endocrinology, Diabetes, and Isotope Therapy, Medical University, Wroclaw, Poland \\ ${ }^{2}$ Department of Endocrinology, Jagiellonian University, Medical College, Krakow, Poland \\ ${ }^{3}$ Department of Endocrinology and Neuroendocrine Tumours, ENETS Centre of Excellence, Department of Pathophysiology \\ and Endocrinology, Medical University of Silesia, Katowice, Poland \\ ${ }^{4}$ Department of Endocrinology, Metabolism, and Internal Diseases, University of Medical Sciences, Poznan, Poland \\ ${ }^{5}$ Department of Internal Medicine, Endocrinology, and Diabetes, Medical University of Warsaw, Poland \\ ${ }^{6}$ Department of Endocrinology, Centre of Postgraduate Medical Education, Warsaw, Poland \\ ${ }^{7}$ Ipsen, Boulogne-Billancourt Cedex, France \\ ${ }^{8}$ Ipsen, Warsaw, Poland
}

\begin{abstract}
Introduction: Patients with acromegaly have substantially reduced quality of life (QoL). This study evaluated QoL in patients with acromegaly treated with lanreotide autogel.

Material and methods: This was a prospective, non-interventional, observational, multi-centre study conducted in Poland (NCT02396966). We included patients with acromegaly, who received treatment with lanreotide autogel $120 \mathrm{mg}$ for $\geq 3$ months and $<3$ years. Patients were assessed approximately every $4-5$ months for two years (six visits). QoL was measured with the Acromegaly Quality of Life Questionnaire (AcroQoL).

Results: Of 152 patients enrolled from November 2014 to May 2018 in 37 centres, 24 were excluded due to major protocol deviations. The results are reported for the study population $(\mathrm{n}=128)$. At baseline, the median [95\% confidence interval $(\mathrm{CI})]$ time from diagnosis was $3.3(2.8,4.2)$ years, and the median time since lanreotide initiation was $13.4(9.9,17.3)$ months. Symptoms of acromegaly were present at baseline in $86 \%$ of patients (headache, $57 \%$; sweating, $58 \%$; joint symptoms, $64 \%$ ); symptoms remained unchanged at two years in $82 \%$ of patients. At baseline, $27 \%$ of patients had hormonal control (growth hormone $<2.5 \mu \mathrm{g} / \mathrm{L}$ and insulin-like growth factor- 1 within the normal range); hormonal control status did not change during the study period in over $81 \%$ of patients. At baseline, $88 \%$ of patients were either very satisfied or satisfied with treatment; treatment satisfaction was unchanged in $62 \%$ of patients over the study period. Mean (95\% CI) AcroQoL scores at baseline were as follows: total, 50.3 (47.3, 53.3); physical dimension, 48.8 (45.2, 52.4); psychological dimension, $51.3(48.2,54.4)$; appearance subdimension, $40.7(37.5,43.8)$; and personal relations subdimension, $62.5(58.8,66.2)$. The psychological appearance subscore improved by 3.8 points $(1.2,6.5)$ over the two years; scores in the remaining dimensions and subdimensions did not change substantially. The total AcroQoL score remained unchanged over the two years, regardless of prior acromegaly treatment, surgery or radiotherapy, hormonal control, or lanreotide dosing interval. No new safety findings were identified.

Conclusions: AcroQoL total scores and physical and psychological subscores remained stable but impaired among patients with longlasting acromegaly treated with lanreotide autogel for two years. The psychological appearance subdimension improved numerically. (Endokrynol Pol 2021; 72 (5): 512-519)
\end{abstract}

Key words: acromegaly; somatostatin analogue; lanreotide; quality of life; AcroQoL

\section{Introduction}

Acromegaly is caused by an excessive secretion of growth hormone $(\mathrm{GH})$, typically by a pituitary adenoma, with a consequent increase in the production of insulin-like growth factor-1 (IGF-1) [1]. The symptoms and complications of acromegaly include acral enlargement, facial disfiguration, arthralgia and arthritis, headache, sleep apnoea, cardiovascular diseases, and disorders of glucose metabolism [1]. Acromegaly worsens both the physical and emotional well-being of patients, and substantially reduces quality of life (QoL) at all disease stages [2]. Patients with acromegaly have much lower scores on generic questionnaires measuring QoL compared to the general population or patients with other chronic diseases, such as asthma, angina, and osteoarthritis [3-5]. The physical aspect of QoL in acromegaly is substantially affected by pain, weak- 
ness, sleep problems, and limitations of daily activities [6]. The psychological aspect of QoL in acromegaly is worsened by a negative perception of one's appearance and difficulties in personal relations [6].

The first-line treatment for most patients with acromegaly is transsphenoidal resection of the adenoma, whereas pharmacological treatment is given to those who have uncontrolled disease after surgery or contraindications to surgery $[7,8]$. Long-acting somatostatin analogues (SSAs), such as octreotide or lanreotide, are the most commonly used medications in patients with acromegaly. The current treatment goals in acromegaly are to reduce or control tumour growth, inhibit GH hypersecretion, and normalize IGF-1 levels, which help control disease complications, decrease mortality, and improve QoL [9]. However, the correlation between clinical severity and the impact of the disease perceived by patients is weak; thus, QoL should be used as an additional outcome to incorporate the patient's perspective [10].

Lanreotide autogel is a long-acting, super-saturated, viscous aqueous formulation of lanreotide injected subcutaneously once every $28-56$ days [11, 12]. In treatment-naïve patients with acromegaly, lanreotide autogel improved their QoL [13]. In this real-world study, we investigated the effect of lanreotide autogel on QoL among patients who had been on this medication for up to three years. Moreover, we related QoL measures to hormonal and clinical variables.

\section{Material and methods}

\section{Study design and setting}

This prospective, non-interventional, observational, multi-centre cohort study was conducted at 37 centres in Poland from November 2014 to May 2018. Participants were enrolled over 18 months. After enrolment, participants were followed up for two years at six visits, approximately once every 4-5 months (V1-6). In this noninterventional study, lanreotide autogel $120 \mathrm{mg}$ was administered and managed within routine care.

\section{Participants}

We included adult patients (aged $>18$ years) with acromegaly, who had received treatment with lanreotide autogel $120 \mathrm{mg}$ for $\geq$ 3 months and $<3$ years. Exclusion criteria were as follows: active participation in any interventional or any other non-interventional acromegaly clinical study or any medical or psychological condition that compromised the ability to give informed consent. Participants were withdrawn if lanreotide autogel $120 \mathrm{mg}$ was stopped or interrupted (i.e. changed for another lanreotide dose or to another SSA). Because this was a non-interventional study, no other withdrawal criteria were specified. Participants were free to withdraw consent at any time. Data were collected up to the time of withdrawal. All patients signed informed consent before enrolment. The study was approved by local Ethics Committees at each study site.

\section{Assessments}

\section{Quality of life}

Quality of life was measured with the Acromegaly Quality of Life (AcroQoL) questionnaire at baseline and then at visits 2, 3, and 6. The AcroQoL questionnaire is a validated disease-specific selfassessment questionnaire designed for use in clinical trials and everyday practice. The AcroQoL questionnaire includes 22 items relating to two dimensions: physical (8 items) and psychological (14 items), the latter being divided into two subdimensions: psychological appearance (7 items) and psychological personal relations (7 items) [6]. Each AcroQoL item is answered on a five-point Likert scale. Moreover, during all study visits, participants gave an overall assessment of treatment satisfaction on a five-point Likert scale (1-5; completely satisfied, rather satisfied, neither satisfied nor dissatisfied, rather dissatisfied, and completely dissatisfied).

\section{Clinical and ancillary assessments}

At baseline, we collected demographic data, date of acromegaly diagnosis, details on current and prior acromegaly treatment (medications, radiation therapy, or surgery), and tumour presence and size (microadenoma $[<10 \mathrm{~mm}]$, macroadenoma $[\geq 10 \mathrm{~mm}$, $<40 \mathrm{~mm}$ ], giant tumour [ $\geq 40 \mathrm{~mm}]$, no tumour). At every visit, we looked for predefined acromegaly symptoms (headache, sweating, joint symptoms, and swelling) and predefined comorbidities (altered carbohydrate metabolism, hypertension, sleep apnoea, heart disease, hypopituitarism, hyperprolactinaemia, hyperthyroidism, hypogonadism, nodular goitre, simplex goitre, and malignant tumour). The dose of lanreotide and the injection interval were recorded at every visit. The tumour size was assessed by magnetic resonance imaging at baseline. The serum concentrations of glucose, GH, and IGF-1 were measured at every visit in local laboratories. Hormonal control was defined as $\mathrm{GH}$ concentrations $<2.5 \mu \mathrm{g} / \mathrm{L}$ and IGF-1 concentrations within age- and sex-specific normal ranges [14]. Adverse events were recorded at every visit; however, because this was a noninterventional study, only related adverse events were reported.

\section{Statistical analysis}

No inferential statistical analyses were planned or performed. All data are presented descriptively overall and by subgroups. The enrolled population was defined as all participants who signed an informed consent form. The study population included participants without major protocol deviations. As the primary endpoint, the AcroQoL total score, AcroQoL dimensions, and subdimensions scores were calculated per visit in the study population. The total AcroQoL score was obtained by adding scores from 22 items with the following formula:

$$
[(X-22) /(110-22)] \times 100
$$

with higher scores indicating better QoL [6]. Secondary endpoints included the control of clinical symptoms, hormonal control, treatment satisfaction, and AcroQoL scores by subgroups. It was planned to enrol 150 patients because this sample size was deemed necessary to describe QoL in the following subgroups: controlled/ uncontrolled disease, with/without prior radiotherapy, with/ without prior surgery.

\section{Results}

\section{Participants}

Of the 152 patients enrolled in the study, 128 were finally included in the study population ( 24 patients were excluded due to major protocol deviations), and 115 completed the study (Fig. 1). The results presented herein are for the study population. 


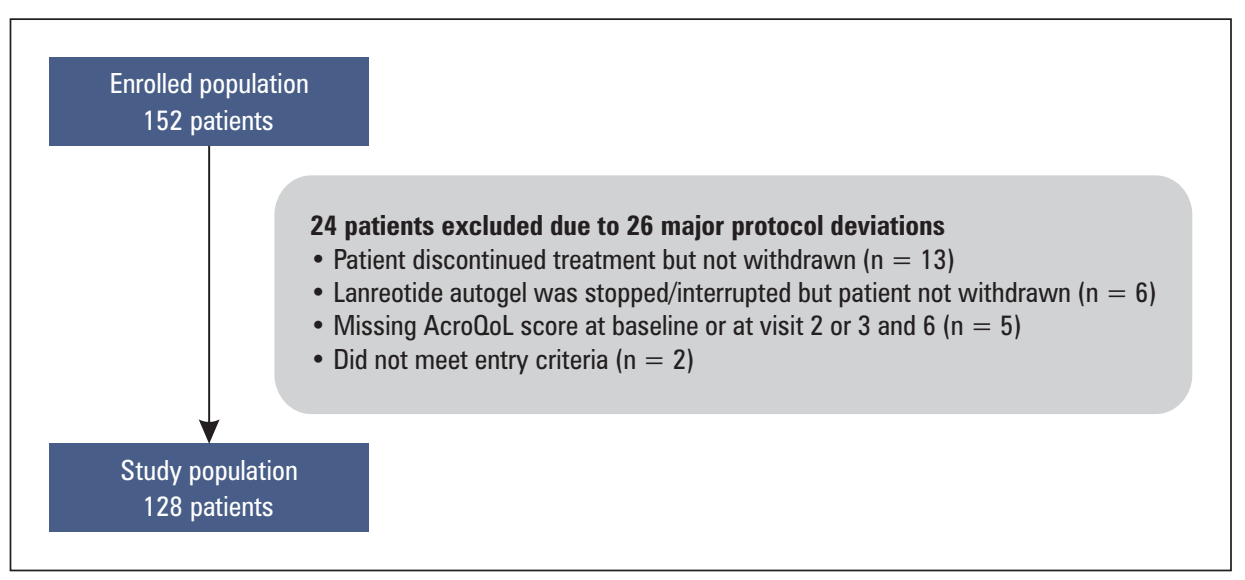

Figure 1. Disposition of patients; AcroQoL — Acromegaly Quality of Life Questionnaire

Patients had a long duration of acromegaly, with a median $(95 \%$ confidence interval $[\mathrm{CI}])$ time from diagnosis of $3.3(2.8,4.2)$ years. At baseline, $110(86 \%)$ patients had symptoms of acromegaly. Most patients had headache (57\%), sweating (58\%), and joint symptoms (64\%). Moreover, the comorbidity burden was high, with most patients having altered carbohydrate metabolism $(56 \%)$, hypertension $(60 \%)$, or nodular goitre (52\%). At baseline, $74 \%$ of patients had visible pituitary tumours on magnetic resonance imaging. Hormonal control was achieved in $27 \%$ of patients at baseline. Other baseline characteristics are presented in Table 1.

Sixty-nine per cent of patients received another acromegaly therapy before lanreotide autogel (including surgery [87.5\%], radiation therapy [13.6\%], and medications [34.1\%]; Tab. 1). At baseline, patients had received lanreotide autogel for a median (95\% CI) of 13.4 (9.9, 17.3) months. The interval of lanreotide autogel $120 \mathrm{mg}$ injections and concomitant medications for acromegaly at baseline are shown in Table 2.

\section{Quality of life and satisfaction with treatment}

The mean (95\% CI) total AcroQoL score was 50.27 (47.25, 53.28) at baseline, and it did not change substantially during the study, with a mean increase from baseline to the end of the study of $1.21(-1.39,3.82)$ points. The mean $(95 \%$ CI) AcroQoL physical dimension score was $48.76(45.16,52.37)$ at baseline, with a mean on-study increase of $1.08(-2.09,4.26)$. The mean (95\% CI) AcroQoL psychological dimension score was 51.30 (48.20, 54.40 ) at baseline, with a mean on-study increase of 1.21 $(-1.43,3.84)$. The mean $(95 \% \mathrm{CI})$ AcroQoL psychological appearance subdimension score was 40.65 (37.47, 43.83) at baseline, with a mean on-study increase of 3.81 $(1.18,6.45)$. The mean $(95 \% \mathrm{CI})$ AcroQoL psychological personal relations subdimension score was 62.53 (58.82, 66.24 ) at baseline, with a mean on-study decrease of -1.38
Table 1. Baseline characteristics in the study population

\begin{tabular}{|c|c|}
\hline & $\begin{array}{l}\text { Study population } \\
\quad(\mathrm{n}=128)\end{array}$ \\
\hline Age, mean $\pm S D$ [years] & $51.6 \pm 13.5$ \\
\hline Women, n (\%) & $71(55.5)$ \\
\hline Height, mean \pm SD $[\mathrm{cm}]$ & $172.5 \pm 10.5$ \\
\hline Weight, mean \pm SD $[\mathrm{kg}]$ & $89.3 \pm 19.6$ \\
\hline $\mathrm{BMI}$, mean $\pm \mathrm{SD}\left[\mathrm{kg} / \mathrm{m}^{2}\right]$ & $30.05 \pm 6.40$ \\
\hline $\begin{array}{l}\text { Time since diagnosis [years], median } \\
\text { (95\% confidence interval) }\end{array}$ & $3.3(2.8,4.2)$ \\
\hline Clinical symptoms, n (\%) & $110(85.9)$ \\
\hline Headache & $73(57.0)$ \\
\hline Sweating & $74(57.8)$ \\
\hline Joint symptoms & $82(64.1)$ \\
\hline Swelling & $53(41.4)$ \\
\hline \multicolumn{2}{|l|}{ Comorbidities, n (\%) } \\
\hline Altered carbohydrate metabolism & $71(55.5)$ \\
\hline Hypertension & $77(60.2)$ \\
\hline Sleep apnoea & 45 (35.2) \\
\hline Heart disease & $26(20.3)$ \\
\hline Hypopituitarism & 35 (27.3) \\
\hline Hyperprolactinaemia & $20(15.6)$ \\
\hline Hyperthyroidism & $7(5.5)$ \\
\hline Hypogonadism & $29(22.7)$ \\
\hline Nodular goitre & $66(51.6)$ \\
\hline Simplex goitre & $8(6.3)$ \\
\hline Malignant tumours & $6(4.7)$ \\
\hline \multicolumn{2}{|c|}{ Therapy before lanreotide autogel, n (\%) } \\
\hline None & $40(31.3)$ \\
\hline Previous surgery & $77(87.5)^{\mathrm{a}}$ \\
\hline Previous radiation therapy & $12(13.6)^{\mathrm{a}}$ \\
\hline
\end{tabular}


Table 1. Baseline characteristics in the study population

\begin{tabular}{lc}
\hline & $\begin{array}{c}\text { Study population } \\
\text { (n = 128) }\end{array}$ \\
\hline Previous medications $(\mathbf{n}=\mathbf{3 0}), \mathbf{n}(\%)$ & $20(66.7)^{\mathrm{b}}$ \\
\hline Octreotide & $1(3.3)^{\mathrm{b}}$ \\
\hline Lanreotide autogel $90 \mathrm{mg}$ & $11(36.7)^{\mathrm{b}}$ \\
\hline Dopamine agonists & \\
\hline Tumour characteristics, $\mathbf{n}(\%)$ & $30(25.9)$ \\
\hline No tumour visible & $27(23.3)$ \\
\hline Microadenoma & $57(49.1)$ \\
\hline Macroadenoma & $2(1.7)$ \\
\hline Giant tumour & $37(51.4)$ \\
\hline GH, $\mathbf{n}(\%)$ & $35(48.6)$ \\
\hline$<2.5 \mu \mathrm{g} / \mathrm{L}$ & $37(40.7)$ \\
\hline$\geq 2.5 \mu \mathrm{g} / \mathrm{L}$ & $54(59.3)$ \\
\hline IGF-1, $\mathbf{n}(\%)$ & $23(27.4)$ \\
\hline Normal range & \\
\hline Outside normal range & \\
\hline Hormonal controlc, $\mathbf{n}(\%)$ & \\
\hline a - of previously treated patients; b - of patients receiving medications; \\
\hline (IGF-1) concentrations within age- and sex-specific normal ranges (as specified \\
by Scacchi and Cavagnini $[14])$
\end{tabular}

$(-4.65,1.89)$. The mean AcroQoL scores are shown in Figure 2.

The total AcroQoL scores remained similar in patients with or without hormonal control, prior acromegaly treatment, prior radiotherapy, and prior surgery (Fig. 3).

\section{Treatment satisfaction}

At baseline, $32 \%$ of patients were very satisfied with the treatment, $56 \%$ were satisfied, $10 \%$ were neither satisfied nor dissatisfied, and $2 \%$ were dissatisfied; none of them were very dissatisfied with the treatment. In $26 \%$
Table 2. Lanreotide autogel dosing interval and treatment duration and concomitant acromegaly medications at baseline

\begin{tabular}{lc}
\hline & $\begin{array}{c}\text { Study population } \\
\text { ( } \mathbf{~ = ~ 1 2 8 ) ~}\end{array}$ \\
\hline Lanreotide autogel dosing interval, $\mathbf{n}(\%)$ & \\
\hline 28 days & $96(75.0)$ \\
\hline 42 days & $22(17.2)$ \\
\hline 56 days & $10(7.8)$ \\
\hline Mode of injection, $\mathbf{n}(\%)$ & \\
\hline Healthcare professional & $124(96.9)$ \\
\hline Patient self-injection & $4(3.1)$ \\
\hline $\begin{array}{l}\text { Duration of lanreotide autogel treatment } \\
\text { (months), median (95\% confidence interval) }\end{array}$ & $13.4(9.9,17.3)$ \\
\hline Concomitant medication, $\mathbf{n}(\%)$ & \\
\hline Dopamine agonist & $29(22.7)$ \\
\hline Octreotide (subcutaneous) & $2(6.5)$ \\
\hline
\end{tabular}

of patients, the satisfaction with treatment improved from baseline to the end of the study, in $62 \%$ of patients it did not change, and $12 \%$ showed a decrease in their satisfaction during the study.

\section{Disease control and change in lanreotide dosing interval}

The hormonal control status did not change in over $81 \%$ of patients, and $33 \%$ had hormonal control at the end of the study. Moreover, there was no change in clinical symptoms in over $82 \%$ of patients from baseline to the end of study.

We found no change in the presence of comorbidities or complications related to acromegaly, including altered carbohydrate metabolism, hypertension, sleep apnoea, heart disease, hypopituitarism, hyperprolactinaemia, hyperthyroidism, simplex goitre, and malignant tumour, in over $91 \%$ of patients. There was also no change in the presence of hypogonadism in over $85 \%$ of
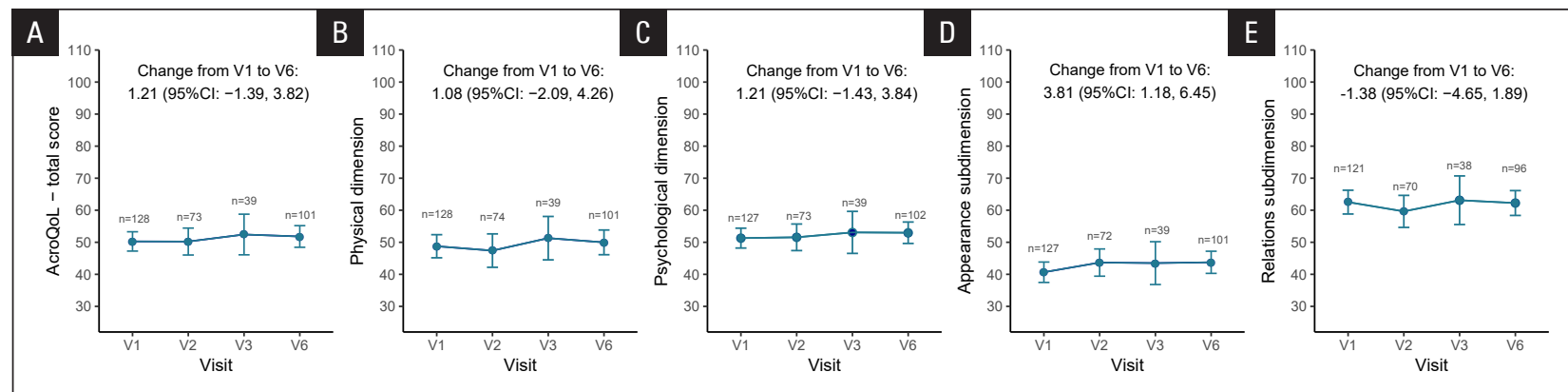

Figure 2. Mean Acromegaly Quality of Life Questionnaire (AcroQoL) scores for the study population at visits (V) 1, 2, 3, and 6. Total AcroQoL scores (A), physical dimension (B), psychological dimension (C), psychological appearance subdimension (D), and psychological personal relations subdimension (E). Data are presented as means [95\% confidence interval (CI)] 


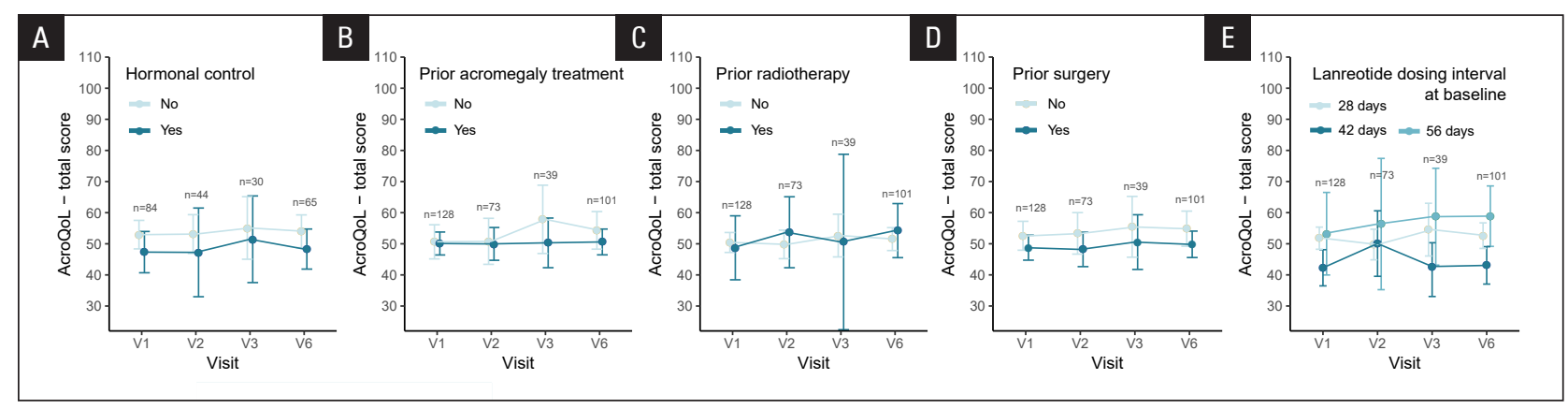

Figure 3. Mean Acromegaly Quality of Life Questionnaire (AcroQoL) scores for the study population at visits (V) 1, 2, 3, and 6 according to hormonal control (A), prior acromegaly treatment (B), prior radiotherapy (C), prior surgery (D), and lanreotide autogel dosing interval (E). Data are presented as means (95\% confidence interval [CI])

patients or nodular goitre in over $83 \%$ of patients. Furthermore, there was no change in the presence of other comorbidities in more than $67 \%$ of patients.

Regarding lanreotide administration and dosing, $84 \%$ of patients in the study population did not change injection frequency from baseline to the end of the study, $63 \%$ of patients received lanreotide autogel once every 28 days, $12 \%$ once every 42 days, and $9 \%$ once every 56 days. No shift in the mode of injection (i.e. self-injection or injection by healthcare professionals) was observed in the study population. Lanreotide autogel was injected by healthcare professionals in $\geq 96 \%$ of patients throughout the study.

\section{Adverse events}

During the study, 15 adverse events associated or coinciding with study drug use (six serious, four non-serious, and five related to product administration) were reported in six participants from the enrolled population. Among the four non-serious adverse events, all were considered to be related to the study medication (diarrhoea [two events], nausea, headache). Among the six serious adverse events, three were considered possibly related to the study drug (diarrhoea, acute cholecystitis, headache). None of the 15 adverse events were associated with a fatal outcome. Three patients died during the study (events not reported as serious adverse events as per study protocol): one death without any details provided, one death for cardiovascular reasons, and one death due to haemorrhagic stroke. No new safety findings or safety issues requiring further investigation were identified in this study.

\section{Discussion}

This real-world, prospective, longitudinal study carried out among patients who had a long duration of acromegaly found that global QoL and its physical and psychological aspects remained stable, whereas the AcroQoL psychological appearance subdimension improved numerically. Most patients were satisfied or very satisfied with the treatment (and no patients were very dissatisfied). Hormonal control and symptoms and complications of acromegaly did not change in most patients over the study. The safety findings were in line with the known safety profile of lanreotide.

Our study provides information on QoL in patients with a long duration of acromegaly. At baseline, the median time since diagnosis was over three years, and the median time of lanreotide autogel treatment was approximately one year, with most patients having received other acromegaly treatments. Approximately $60 \%$ of the 128 patients had headache and joint symptoms, which can reduce QoL in those with acromegaly [15]. Indeed, previous research showed that patients with acromegaly and joint pain had a substantially lower QoL (AcroQoL score, 59) than those without joint pain (AcroQoL, 76) [16]. Similarly, patients with acromegaly and spinal joint complaints scored lower than those without these comorbidities on several QoL questionnaires, including AcroQoL ( 61 vs. $\sim 71$ points) [17]. Headaches are also associated with decreased QoL or no improvement in QoL among patients with acromegaly [18, 19].

Overall, the QoL in our cohort was substantially reduced, with a mean total AcroQoL score of $\sim 50$, which is lower than healthy people and patients with acromegaly from previous studies. For example, in a study from Romania, the mean total AcroQoL was $\sim 76$ in healthy people and 61 in patients with acromegaly [20]. In another study, among patients with a "severe" reduction of QoL, the mean total AcroQoL was higher than in our study (AcroQoL, 57) [3]. Yet, a similar QoL to our cohort was observed among patients with long-lasting acromegaly ( $>10$ years) and a significant disease burden (AcroQoL, 54 ), and it did not change over five years [21]. Similarly, after treatment of GH excess, Biermasz et al. found that 118 patients with 
acromegaly had persistently decreased QoL despite biochemical cure [22]. These findings suggest that long-lasting acromegaly causes a sustained reduction of QoL, despite disease-specific treatment.

Although the total AcroQoL score did not change substantially in our cohort, the improvement in the psychological dimension might be important to patients with acromegaly. Indeed, in a study that used AcroQoL to compare QoL between patients with acromegaly and people with obesity, the psychological appearance subdimension was the most reduced subscale in patients with acromegaly [23].

In a study among 90 treatment-naïve patients with acromegaly (mean disease duration of four months), who received lanreotide autogel $120 \mathrm{mg}$, Caron et al. found an improvement in all AcroQoL scores after one year of treatment (mean change of $\sim 5 \%$ on the personal relations subdimension, $\sim 10 \%$ in the total, physical, and psychological dimensions; and $\sim 15 \%$ on the appearance subdimension) [24]. However, the difference in the characteristics of participants (i.e. the duration of acromegaly and type of treatment) could explain why the improvement in QoL was greater in the study by Caron et al. than in our study.

An improvement in QoL was also observed among 53 treatment-naïve patients with acromegaly, who received lanreotide autogel $120 \mathrm{mg}$ (QoL assessed with the Nottingham questionnaire) [13]. In contrast, we found no change in QoL among patients in our cohort who had been on lanreotide treatment for up to three years (median of approximately one year). Meanwhile, a small study showed that AcroQoL scores improved during four years in patients who received octreotide [18]; however, this was a retrospective study that could have had selection bias (i.e. selective retainment of patients who perceived improvement). In line with this assumption, a 24-week prospective study found a modest improvement of QoL on octreotide only in the psychological subdimension, similarly to our study [25].

Similarly to most previous reports, we did not find that the hormonal status affected the QoL [26]. QoL remained stable in our cohort regardless of baseline hormonal control status, prior surgery, prior radiotherapy, or lanreotide dosing interval. Likewise, Matta et al. found that QoL in patients with a mean duration of acromegaly of 10 years was similar regardless of hormonal control (AcroQoL, 60) [27]. However, another study showed that QoL was higher (AcroQoL, $\sim 61-71$ ) among patients with long-lasting acromegaly ( $>10$ years) who achieved hormonal control than that observed in our cohort [17]. Thus, the importance of hormonal control of acromegaly for QoL requires further investigation.
Our study was limited by a heterogeneous sample (disease duration, previous treatments), and thus, the effect of lanreotide autogel treatment on QoL could be obscured by confounding factors. However, our study provides important real-world evidence that may be more relevant to clinical practice than the findings from clinical trials, in which only selected patients, typically shortly after diagnosis, are followed for a limited time. Our cohort of patients reflected, for example, the high prevalence of comorbidities that are typically observed among patients with acromegaly [28]. The available evidence indicates that, in the long-term, acromegaly causes considerable problems that may not be directly related to the biological aspects of the disease. These problems include everyday challenges like a negative body image and major life changes, such as employment issues [2,29]. Our study presents a picture of the most common symptoms and complications of acromegaly among patients in Poland. As in previous studies, most patients had joint symptoms, headache, and sweating [30-32]. Arterial hypertension, altered glucose metabolism, and nodular goitre were also frequent $[33,34]$.

It is worth mentioning that despite the lack of improvement in symptoms and QoL, overall the patients in this study were kept on the same therapy. A potential reason is that at the time of the study, there were only two long-acting SSAs available for acromegaly treatment in Poland: octreotide LAR and lanreotide autogel. Other treatment options, such as pasireotide (a somatostatin analogue) and pegvisomant (a GH receptor antagonist), were not reimbursed at the time of the current study. Nonetheless, further symptomatic treatment, such as physiotherapy, offered in addition to disease-specific medications like lanreotide, could help improve QoL in patients with long-lasting acromegaly. Indeed, an interdisciplinary approach of treating the various comorbidities and symptoms present in patients with acromegaly seems to be more effective than disease-specific treatment alone [35,36]. Evidence-based guidelines for the treatment of acromegaly complications (including cardiovascular, endocrine, metabolic, and oncologic comorbidities, sleep apnoea, and bone and joint disorders) have recently been produced by the Acromegaly Consensus Group, and they could assist in improving QoL among patients with acromegaly [37].

\section{Conclusions}

We found that QoL remains stable in patients with long-lasting acromegaly and a substantial disease burden during two years of treatment with lanreotide autogel. Despite good control of symptoms throughout the study, the total AcroQoL scores, including those of 
the AcroQoL physical and psychological dimensions, were unchanged. However, scores on the AcroQoL psychological appearance subdimension improved numerically, which is promising because this subscale is often significantly reduced in patients with acromegaly. Moreover, most patients remained satisfied with lanreotide autogel treatment during the study. Further studies into whether other interventions (like physiotherapy or the targeted treatment of acromegaly complications) combined with lanreotide autogel therapy improve QoL in patients with long-lasting acromegaly are warranted.

\section{Disclosures}

A.H., M.Ba. - employees of Ipsen; M.Bo., A.H.D., B.K.K., W.Z. - honoraria from Novartis, Pfizer, Ipsen, Recordati, Merck; M.R. - honoraria from Novartis, Pfizer, Ipsen, Berlin Chemie, Genzyme, Merck; P.W. - honoraria from Novartis, Pfizer, Ipsen, Berlin Chemie.

\section{Acknowledgements}

The authors thank all patients involved in the study and their caregivers, investigators, and research staff in participating institutions.

The authors thank Rafał Szot and Agnieszka Linkiewicz-Zegan (Proper Medical Writing, Warsaw, Poland) for their help with the preparation of the manuscript. Medical writing assistance was sponsored by Ipsen in accordance with Good Publication Practice guidelines.

\section{References}

1. Melmed S. Medical progress: Acromegaly . N Engl J Med . 2006; 355(24): 2558-2573, doi: 10.1056/NEJMra062453, indexed in Pubmed: 17167139.

2. Gurel $\mathrm{MH}$, Bruening PR, Rhodes $\mathrm{C}$, et al. Patient perspectives on the impact of acromegaly: results from individual and group interviews. Patient Prefer Adherence. 2014; 8: 53-62, doi: 10.2147/PPA.S56740, indexed in Pubmed: 24453479.

3. Rowles SV, Prieto L, Badia X, et al. Quality of life (QOL) in patients with acromegaly is severely impaired: use of a novel measure of QOL: acromegaly quality of life questionnaire. J Clin Endocrinol Metab. 2005; 90(6): 3337-3341, doi: 10.1210/jc.2004-1565, indexed in Pubmed: 15755865

4. Szcześniak DM, Jawiarczyk-Przybyłowska A, Matusiak $€$, et al. Is there any difference in acromegaly and other chronic disease in quality of life and psychiatric morbidity? Endokrynol Pol. 2017; 68(5): 524-532, doi: 10.5603/EP.a2017.0044, indexed in Pubmed: 28879648.

5. Kauppinen-Mäkelin R, Sane T, Sintonen H, et al. Quality of life in treated patients with acromegaly. J Clin Endocrinol Metab. 2006; 91(10): 3891-3896, doi: 10.1210/jc.2006-0676, indexed in Pubmed: 16849407.

6. Badia X, Webb SM, Prieto L, et al. Acromegaly Quality of Life Questionnaire (AcroQoL). Health Qual Life Outcomes. 2004; 2: 13, doi: 10.1186/1477-7525-2-13, indexed in Pubmed: 14987332

7. Giustina A, Chanson P, Kleinberg D, et al. Acromegaly Consensus Group. Expert consensus document: A consensus on the medical treatment of acromegaly. Nat Rev Endocrinol. 2014; 10(4): 243-248, doi: 10.1038/nrendo.2014.21, indexed in Pubmed: 24566817.

8. Bolanowski M, Ruchała M, Zgliczyński W, et al. Diagnostics and treatment of acromegaly - updated recommendations of the Polish Society of Endocrinology. Endokrynol Pol. 2019; 70(1): 2-18, doi: 10.5603/EP.a2018.0093, indexed in Pubmed: 30843181.

9. Melmed S, Bronstein MD, Chanson P, et al. A Consensus Statement on acromegaly therapeutic outcomes. Nat Rev Endocrinol. 2018; 14(9): 552-561, doi: 10.1038/s41574-018-0058-5, indexed in Pubmed: 30050156.
10. Crespo I, Valassi E, Webb SM. Update on quality of life in patients with acromegaly. Pituitary. 2017; 20(1): 185-188, doi: 10.1007/s11102-016-0761-y, indexed in Pubmed: 27730455.

11. Melmed S, Cook D, Schopohl J, et al. Rapid and sustained reduction of serum growth hormone and insulin-like growth factor- 1 in patients with acromegaly receiving lanreotide Autogel therapy: a randomized, placebo-controlled, multicenter study with a 52 week open extension. Pituitary. 2010; 13(1): 18-28, doi: 10.1007/s11102-009-0191-1, indexed in Pubmed: 19639415.

12. Bevan JS, Newell-Price J, Wass JAH, et al. Home administration of lanreotide Autogel by patients with acromegaly, or their partners, is safe and effective. Clin Endocrinol (Oxf). 2008; 68(3): 343-349, doi: 10.1111/j .1365-2265.2007.03044.x, indexed in Pubmed: 17892497.

13. Lombardi G, Minuto F, Tamburrano G, et al. Efficacy of the new long-acting formulation of lanreotide (lanreotide Autogel) in somatostatin analogue-naive patients with acromegaly. J Endocrinol Invest. 2009; 32(3): 202-209, doi: 10.1007/BF03346453, indexed in Pubmed: 19542735.

14. Scacchi M, Cavagnini F. Acromegaly. Pituitary. 2006; 9(4): 297-303, doi: 10.1007/s11102-006-0409-4, indexed in Pubmed: 17077948.

15. Szcześniak D, Jawiarczyk-Przybyłowska A, Rymaszewska J. The quality of life and psychological, social and cognitive functioning of patients with acromegaly. Adv Clin Exp Med. 2015; 24(1): 167-172, doi: 10.17219/acem/38156, indexed in Pubmed: 25923102.

16. Miller A, Doll H, David J, et al. Impact of musculoskeletal disease on quality of life in long-standing acromegaly. Eur J Endocrinol. 2008; 158(5): 587-593, doi: 10.1530/EJE-07-0838, indexed in Pubmed: 18426816.

17. Wassenaar MJE, Biermasz NR, Kloppenburg M, et al. Clinical osteoarthritis predicts physical and psychological QoL in acromegaly patients. Growth Horm IGF Res. 2010; 20(3): 226-233, doi: 10.1016/j. ghir.2010.02.003, indexed in Pubmed: 20194043.

18. Mangupli R, Camperos P, Webb SM. Biochemical and quality of life responses to octreotide-LAR in acromegaly. Pituitary. 2014; 17(6): 495-499, doi: 10.1007/s11102-013-0533-x, indexed in Pubmed: 24178448.

19. Dimopoulou C, Athanasoulia AP, Hanisch E, et al. Clinical characteristics of pain in patients with pituitary adenomas. Eur J Endocrinol. 2014; 171(5): 581-591, doi: 10.1530/EJE-14-0375, indexed in Pubmed: 25117460.

20. Solomon E, Brănișteanu D, Dumbravă A, et al. Executive functioning and quality of life in acromegaly. Psychol Res Behav Manag. 2019; 12: 39-44, doi: 10.2147/PRBM.S183950, indexed in Pubmed: 30655710.

21. Kyriakakis N, Lynch J, Gilbey SG, et al. Impaired quality of life in patients with treated acromegaly despite long-term biochemically stable disease: Results from a 5-years prospective study. Clin Endocrinol (Oxf). 2017; 86(6): 806-815, doi: 10.1111/cen.13331, indexed in Pubmed: 28316090.

22. Biermasz NR, van Thiel SW, Pereira AM, et al. Decreased quality of life in patients with acromegaly despite long-term cure of growth hormone excess. J Clin Endocrinol Metab. 2004; 89(11): 5369-5376, doi: 10.1210/jc.2004-0669, indexed in Pubmed: 15531483.

23. Webb SM, Badia X, Surinach NL, et al. Spanish AcroQol Study Group. Validity and clinical applicability of the acromegaly quality of life questionnaire, AcroQoL: a 6-month prospective study. Eur J Endocrinol. 2006; 155(2): 269-277, doi: 10.1530/eje.1.02214, indexed in Pubmed: 16868140.

24. Caron PJ, Bevan IS, Petersenn S, et al. PRIMARYS Investigators Group. Effects of lanreotide Autogel primary therapy on symptoms and quality-of-life in acromegaly: data from the PRIMARYS study. Pituitary. 2016; 19(2): 149-157, doi: 10.1007/s11102-015-0693-y, indexed in Pubmed: 26603536

25. Chin SO, Chung CH, Chung YS, et al. Change in quality of life in patients with acromegaly after treatment with octreotide LAR: first application of AcroQoL in Korea. BMJ Open. 2015; 5(6): e006898, doi: 10.1136/bmjopen-2014-006898, indexed in Pubmed: 26063564.

26. Geraedts VJ, Andela CD, Stalla GK, et al. Predictors of Quality of Life in Acromegaly: No Consensus on Biochemical Parameters. Front Endocrinol (Lausanne). 2017; 8: 40, doi: 10.3389/fendo.2017.00040, indexed in Pubmed: 28316591.

27. Matta MP, Couture E, Cazals L, et al. Impaired quality of life of patients with acromegaly: control of GH/IGF-I excess improves psychological subscale appearance. Eur J Endocrinol. 2008; 158(3): 305-310, doi: 10.1530/EJE-07-0697, indexed in Pubmed: 18299462

28. Lesén E, Granfeldt D, Houchard A, et al. Comorbidities, treatment patterns and cost-of-illness of acromegaly in Sweden: a register-linkage population-based study. Eur J Endocrinol. 2017; 176(2): 203-212, doi: 10.1530/EJE-16-0623, indexed in Pubmed: 27932528.

29. Strasburger CJ, Karavitaki N, Störmann S, et al. Patient-reported outcomes of parenteral somatostatin analogue injections in 195 patients with acromegaly. Eur J Endocrinol. 2016; 174(3): 355-362, doi: 10.1530/EJE-15-1042, indexed in Pubmed: 26744896.

30. Jadresic A, Banks LM, Child DF, et al. The acromegaly syndrome. Relation between clinical features, growth hormone values and radiological characteristics of the pituitary tumours. Q J Med. 1982; 51(202): 189-204, indexed in Pubmed: 7111679. 
31. Lugo G, Pena L, Cordido F. Clinical manifestations and diagnosis of acromegaly. Int J Endocrinol. 2012; 2012: 540398, doi: 10.1155/2012/540398, ndexed in Pubmed: 22518126.

32. Caron P, Brue T, Raverot G, et al. Signs and symptoms of acromegaly at diagnosis: the physician's and the patient's perspectives in the ACRO-PO LIS study. Endocrine. 2019; 63(1): 120-129, doi: 10.1007/s12020-018-1764-4, indexed in Pubmed: 30269264.

33. Gasperi M, Martino E, Manetti L, et al. Acromegaly Study Group of the Italian Society of Endocrinology. Prevalence of thyroid diseases in patients with acromegaly: results of an Italian multi-center study. J Endocrinol Invest. 2002; 25(3): 240-245, doi: 10.1007/BF03343997, indexed in Pubmed: 11936466.

34. Caron P, Brue T, Raverot G, et al. Signs and symptoms of acromegaly at diagnosis: the physician's and the patient's perspectives in the ACRO-PO-
LIS study. Endocrine. 2019; 63(1): 120-129, doi: 10.1007/s12020-018-1764-4, indexed in Pubmed: 30269264

35. Adelman DT, Liebert KJp, Nachtigall LB, et al. Acromegaly: the disease, its impact on patients, and managing the burden of long-term treatment. Int J Gen Med. 2013; 6: 31-38, doi: 10.2147/IJGM.S38594, indexed in Pubmed: 23359786.

36. Plunkett C, Barkan AL. The care continuum in acromegaly: how patients, nurses, and physicians can collaborate for successful treatment experiences. Patient Prefer Adherence. 2015; 9: 1093-1099, doi: 10.2147/PPA. S84887, indexed in Pubmed: 26251582

37. Giustina A, Barkan A, Beckers A, et al. A Consensus on the Diagnosis and Treatment of Acromegaly Comorbidities: An Update. J Clin Endocrinol Metab. 2020; 105(4), doi: 10.1210/clinem/dgz096, indexed in Pubmed: 31606735. 